\title{
An Elegant Method of Analysis for the BJT Amplifiers using Floating Admittance Matrix
}

\author{
Sanjay Kumar Roy ${ }^{1}$, Kamal Kumar Sharma ${ }^{2}$, Brahmadeo Prasad Singh ${ }^{3}$ \\ ${ }^{1} \mathrm{PhD}$. Scholar at Lovely Professional University and Head (Power Distribution), OMQ Division, \\ TATA Steel Ltd, India. corresponding author: sanjay.roy@tatasteel.com \\ ${ }^{2}$ Professor, Electronics and Communication Engineering, \\ Lovely Professional University, Phagwara, Punjab, India. \\ ${ }^{3}$ Professor Adjunct, Electronics and Communication Engineering, \\ Netaji Subhas University of Technology, New Delhi, India.
}

\begin{abstract}
The Floating Admittance Matrix (FAM) is an elegant, neat, illustrative, and simplified technique for analyzing all configurations of the BJT amplifiers, starting with the maneuvering of the FAM of the phase-splitter circuit. The conventional analysis method requires a small-signal equivalent circuit, and then conventional tools, either KCL, KVL, or Thevenin, Norton, etc., are used for the analysis. The researcher has to guess which conventional tool suites better than the other for any particular circuit, whether active or passive. The proposed technique is equally ell useful for all circuits. In the FAM method, once the device matrix is known rest of the circuit can be embedded in it by inspection. The sum property of this matrix provides a check to know whether FAM has been written correctly to proceed further.
\end{abstract}

Keywords-BJT, CB， CE, CC, Floating Admittance Matrix (FAM), hybrid-model, hybrid- $\pi$ model, T-model.

\section{NTRODUCTION}

There are several methods of mathematical modelling available in the literature based on the equivalent circuit approach. Chirlian [1] suggested a very general approach; wherein any three-terminal devices can fit in. Sedra [2]-[6] have provided unique approaches to the equivalent circuit of the BJTs. We have to select the proper mathematical modelling scheme under given a constraint to achieve the best result with ease. The nullor [7]-[8] and admittance matrix [9] methods have been used in the symbolic form extensively in the past. We have suggested an elegant mathematical modelling approach for both active devices and passive circuits, called the floating admittance matrix model. As the word spelt floating, it does not have any reference terminal in the analysis and design of any circuit, whether active or passive or mixed of the two. The floating admittance matrix (FAM) model has been developed for the BJT to demonstrate the simplicity of the method over other conventional techniques. The outcome of the developed mathematical models has been used for any amplifier configuration that corroborates the result obtained in the available literature, overriding in the simplicity.

The background of the article forms the basis for arriving at the solution of circuits containing two or more than two BJTs or even MOSFETs and BJTs in different configurations. It is likely that one can make mistakes in putting the smallsignal equivalent of the entire complicated circuit, which can lead to an erroneous solution. Additionally, a researcher must be able to guess for a particular circuit which conventional tools KCL, KVL, Thevenin, and Norton etc., becomes suitable for arriving at the solution with ease. Solution of even passive circuits such as twin- $\mathrm{T}$, bridge-T, and lattice networks run into pages using conventional tools. On the contrary, the same FAM is used for all types of circuits, whether simple or complicated, taking the help of matrix partitioning in the case of complicated circuits.

The history of the development of FAM using hybrid small-signal equivalent circuits of the devices and its applications in deriving all types of transfer and self-node functions of any amplifier configuration dates back to 1978 . However, we developed the FAM of the active device using recent trends in the small-signal model of the devices i.e. Tand hybrid- $\pi$, and used it in arriving at the solutions of all 
types of transfer functions of different configurations of the amplifier, with ease, in this article.

We aim to derive all types of transfer functions of all BJT configurations, starting with a properly biased ac circuit of a phase-splitter amplifier shown in Fig.1.

Before we start the analysis of any configurations of the BJT amplifier, we develop the floating admittance matrix in resistance $\left(\boldsymbol{r}_{e}\right)$, in hybrid- $\pi$, and in hybrid $\left(h_{e}\right)$ models of the BJT.

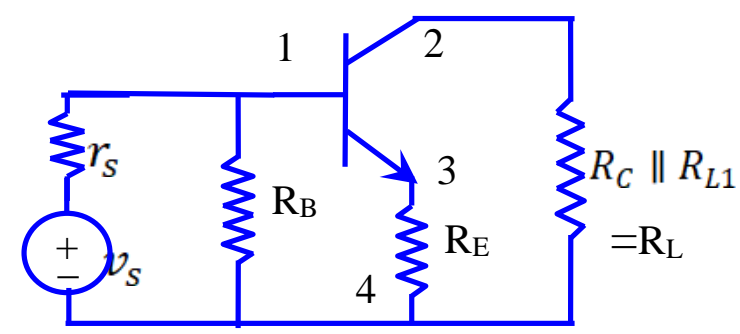

Fig. 1 BJT Phase-Splitter Amplifier

Among these models, the hybrid- $\pi$ model is versatile. For this reason, we take up the development of the floating admittance matrix starting with T-model, then in $\boldsymbol{r}_{\boldsymbol{\pi}}$, and finally in $\boldsymbol{h}_{\boldsymbol{e}}$ model. To indicate the superiority of the floating admittance matrix approach over the convention method of analysis, we derive all types of transfer functions of the amplifiers for a comparison.

\section{MODEL DEVELOPMENT OF BJT}

\section{A. FAM of BJT in T- Model}

Figure 2 (a) simplifies as Fig. 2 (b) with forward-biased dynamic resistance of the base-emitter junction diode along with a controlled current source $\beta i_{b}$ [1]-[6].

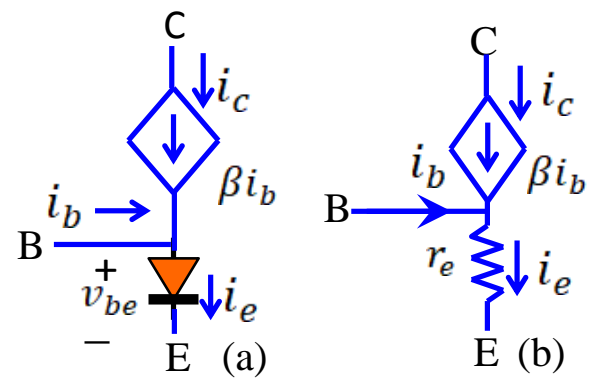

Fig. 2 Common emitter configuration of the BJT

The input and output side of the BJT in Fig. 2 (b) has to be isolated for the ease in the analysis. The isolation of input side to output in Fig. 2 (b) is achieved using the generator splitting theorem as in Fig. 3.

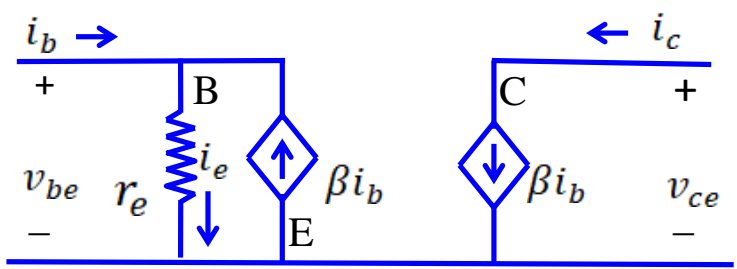

Fig. 3 Splitting the current source

The currents at the base point are the same before and after splitting the current source in Fig. 3. The algebraic sum of current at the base point $=i_{b}+\beta i_{b}=(1+\beta) i_{b}$; which is equal to the emitter current $i_{e}$ that flows through the dynamic resistance of the diode $r_{e}$ and then the controlled source $\beta i_{b}$ is left open, resulting Fig. 4.

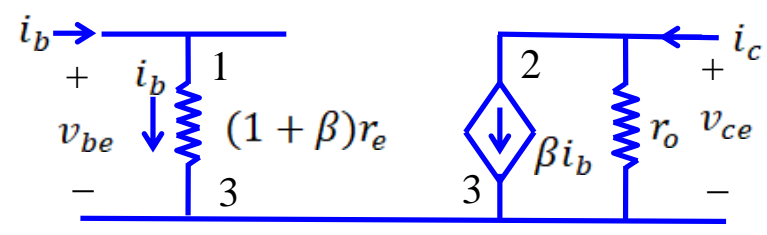

Fig. 4 Input side and output sides isolated

The input resistance is seen through the base terminal of the $\mathrm{CE}$ configuration in Fig. 4 results as;

$$
\begin{aligned}
& v_{b e}=r_{e} i_{e}=r_{e}(1+\beta) i_{b} \\
& r_{i n(C E)}=\frac{v_{b e}}{i_{b}}=\frac{r_{e}(1+\beta) i_{b}}{i_{b}}=(1+\beta) r_{e}=r_{\pi}
\end{aligned}
$$

The input resistance in $\mathrm{CB}$ configuration is obtained by looking into the input of the amplifier through the emitter terminal and is given as;

$$
r_{i n(C B)}=\frac{v_{e b}}{i_{e}}=r_{e}
$$

The reverse-biased collector-base junction offers very large output resistance $r_{o}$, obtained using the Early voltage equation. This $r_{o}$ is included in Fig. 4 to complete the largesignal equivalent circuit of the BJT. The derivation of the floating admittance matrix of the BJT assumes three digits 1, 2 , and 3, assigning it to the base, the collector, and the emitter terminals, respectively. Hence, all terminal voltages and currents are expressed using these three digits in further analysis. Form Fig. 4,

$$
\begin{aligned}
i_{b}=i_{1} & =\frac{1}{(1+\beta) r_{e}} v_{1}+(0) v_{2}-\frac{1}{(1+\beta) r_{e}} v_{3} \\
& =\frac{g_{e}}{(1+\beta)} v_{1}+(0) v_{2}-\frac{g_{e}}{(1+\beta)} v_{3} \\
i_{c}=i_{2} & =\beta i_{b}+g_{o}\left(v_{c e}\right)=\beta i_{1}+g_{o}\left(v_{c}-v_{e}\right) \\
& =\beta\left\{\frac{g_{e}}{(1+\beta)} v_{1}-\frac{g_{e}}{(1+\beta)} v_{3}\right\}+g_{o}\left(v_{2}-v_{3}\right) \\
i_{c}=i_{2} & =\frac{\beta g_{e}}{1+\beta} v_{1}-\frac{\beta g_{e}}{1+\beta} v_{3}+g_{o}\left(v_{2}-v_{3}\right) \\
& =g_{e} v_{1}+g_{o} v_{2}-g_{e} v_{3}+g_{o}\left(-v_{3}\right) \\
i_{2}=i_{c} & =g_{e} v_{1}+g_{o} v_{2}-\left\{g_{e}+g_{o}\right\} v_{3}
\end{aligned}
$$

Applying KCL, $i_{1}+i_{2}+i_{3}=0$,

$$
\begin{aligned}
& i_{3}=-i_{1}-i_{2} \\
& =-\frac{g_{e}}{(1+\beta)} v_{1}+\frac{g_{e}}{(1+\beta)} v_{3}-g_{e} v_{1}-g_{o} v_{2}+\left\{g_{e}+g_{o}\right\} v_{3} \\
& i_{3}=-\left\{g_{e}+\frac{g_{e}}{1+\beta}\right\} v_{1}-g_{o} v_{2}+\left\{g_{e}+\frac{g_{e}}{1+\beta}+g_{o}\right\} v_{3}
\end{aligned}
$$

Arranging $\mathrm{Eq} \mathrm{(3)} \mathrm{to} \mathrm{Eq}$ (5) in the matrix form yields the floating admittance matrix [10]-[14] of BJT in $g_{e}=1 / r_{e}$ model as; 
$\left[\begin{array}{l}i_{1} \\ i_{2} \\ i_{3}\end{array}\right]=\left[\begin{array}{ccc}\frac{g_{e}}{1+\beta} & 0 & -\frac{g_{e}}{1+\beta} \\ g_{e} & g_{o} & -g_{e}-g_{o} \\ \frac{g_{e}}{1+\beta}-g_{e} & -g_{o} & \frac{g_{e}}{1+\beta}+g_{e}+g_{0}\end{array}\right]\left[\begin{array}{l}v_{1} \\ v_{2} \\ v_{3}\end{array}\right]$

The simplified floating admittance matrix of the BJT in $r_{e}$

model is

$$
\left[\begin{array}{ccc}
g_{e}^{\prime} & 0 & -g_{e}^{\prime} \\
g_{e} & g_{o} & -g_{e}-g_{o} \\
g_{e}^{\prime}-g_{e} & -g_{o} & g_{e}^{\prime}+g_{e}+g_{o}
\end{array}\right]
$$

here $r_{e}=\frac{v_{b e}}{i_{e}}=\frac{\frac{v_{b e}}{i_{c}}}{\frac{i_{e}}{i_{c}}}=\left(\frac{v_{b e}}{i_{c}}\right)\left(\frac{i_{c}}{i_{e}}\right)=\frac{a}{g_{m}}$,

$$
g_{e}^{\prime}=\frac{g_{e}}{(1+\beta)}, g_{e}=\frac{1}{r_{e}}, g_{o}=\frac{1}{r_{o}}
$$

\section{B. FAM OF BJT IN $\mathbf{r}_{\mathbf{m}}$ MODEL}

Fig. 5 is the simplified circuit of hybrid- $\pi$ model of the BJT [2]-[3]. The floating admittance matrix of hybrid- $\pi$ can be obtained by analyzing Fig. 5 .

From Fig. 5,

$i_{1}=g_{\pi} v_{\pi}=g_{\pi}\left(v_{1}-v_{3}\right)=g_{\pi} v_{1}+(0) v_{2}-g_{\pi} v_{3}$

$i_{2}=g_{m} v_{\pi}+g_{o}\left(v_{2}-v_{3}\right)=g_{m}\left(v_{1}-v_{3}\right)+g_{o}\left(v_{2}-v_{3}\right)$

$=g_{m} v_{1}+g_{o} v_{2}-\left(g_{m}+g_{o}\right) v_{3}$

$i_{3}=-g_{\pi} v_{1}+g_{\pi} v_{3}-g_{m} v_{1}-g_{o} v_{2}+\left(g_{m}+g_{o}\right) v_{3}$

$=-\left(g_{\pi}+g_{m}\right) v_{1}-g_{0} v_{2}+\left(g_{\pi}+g_{m}+g_{0}\right) v_{3}$

Arranging (9)-(11) yields the matrix of hybrid- $\pi$ as;

$\left[\begin{array}{l}i_{1} \\ i_{2} \\ i_{3}\end{array}\right]=\left[\begin{array}{ccc}g_{\pi} & 0 & -g_{\pi} \\ g_{m} & g_{o} & -g_{m}-g_{o} \\ -g_{\pi}-g_{m} & -g_{o} & g_{\pi}+g_{m}+g_{o}\end{array}\right]\left[\begin{array}{l}v_{1} \\ v_{2} \\ v_{3}\end{array}\right]$

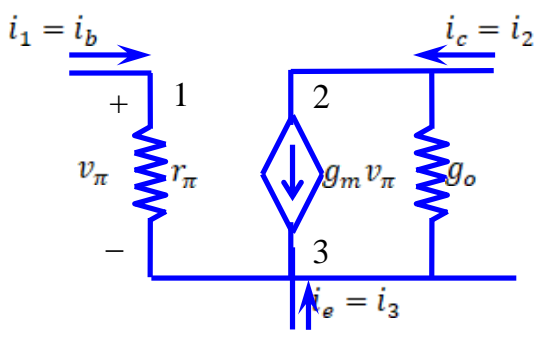

Fig. 5 Hybrid- $\pi$ equivalent circuit

C. FAM OF BJT IN H-PARAMETER MODEL

For the development of the floating admittance matrix of the BJT in the h-parameter model [1]-[5], Fig. 6 is drawn and from this figure,

$v_{1}-v_{3}=h_{i} i_{1}-h_{r}\left(v_{2}-v_{3}\right)$

$i_{1}=\frac{v_{1}}{h_{i}}-\frac{v_{3}}{h_{i}}+\frac{h_{r}}{h_{i}} v_{2}-\frac{h_{r}}{h_{i}} v_{3}$

$=g_{i} v_{1}-g_{i} v_{3}+g_{t} h_{7} v_{2}-g_{t} h_{7} v_{3}$

$i_{1}=g_{i} v_{1}+(0) v_{2}-g_{i} v_{3}$

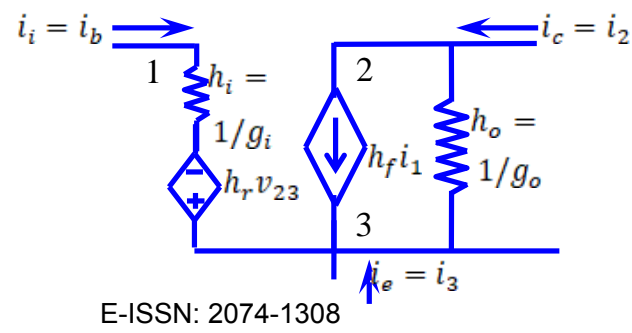

Fig. 6 h-parameter model of the BJT

$$
\begin{aligned}
& i_{2}=h_{f} i_{1}+g_{o}\left(v_{2}-v_{3}\right) \\
& =h_{f} g_{i} v_{1}-h_{f} g_{i} v_{3}+g_{o}\left(v_{2}-v_{3}\right) \\
& =g_{m} v_{1}-g_{m} v_{3}+g_{o}\left(v_{2}-v_{3}\right) \\
& i_{2}=g_{m} v_{1}+g_{o} v_{2}-\left(g_{m}+g_{o}\right) v_{3} \\
& i_{3}=-i_{1}-i_{2} \\
& =-\left(g_{i}+g_{m}\right) v_{1}-g_{o} v_{2}+\left(g_{i}+g_{m}+g_{o}\right) v_{3}
\end{aligned}
$$

Arranging Eqs [13]-[15] yields the floating admittance matrix as;

$\left[\begin{array}{l}i_{1} \\ i_{2} \\ i_{3}\end{array}\right]=\left[\begin{array}{ccc}g_{i} & 0 & -g_{i} \\ g_{m} & g_{o} & -g_{m}-g_{o} \\ -g_{i}-g_{m} & -g_{o} & g_{i}+g_{m}+g_{o}\end{array}\right]\left[\begin{array}{l}v_{1} \\ v_{2} \\ v_{3}\end{array}\right]$

The floating admittance matrix of BJT in $r_{e}, r_{\pi}$, and $h_{e}$ Models are available in Eqs (7), (12), and (16), respectively.

\section{BJT PHASE SPLITTER AMPLIFIER}

To analyze the BJT phase-splitter circuit of Fig. 1, the floating admittance matrix of the BJT in the hybrid- $\pi$ model of Eq (12) is used as it is versatile. The overall floating admittance matrix of active and passive elements present in Fig. 1 results [10][17] as;

$\left[\begin{array}{cccc}1 & 2 & 3 & 4 \\ g_{\pi}+g_{s}+G_{B} & 0 & -g_{\pi} & -g_{s}-G_{B} \\ g_{m} & g_{o}+G_{L} & -g_{m}-g_{o} & -G_{L} \\ -g_{\pi}-g_{m} & -g_{o} & g_{\pi}+g_{m}+g_{o}+G_{E} & -G_{E} \\ -g_{s}-G_{B} & -G_{L} & -G_{E} & g_{s}+G_{B}+G_{E}+G_{L}\end{array}\right]\left[\begin{array}{l}1 \\ 1 \\ 2 \\ 3 \\ 4\end{array}\right]$

where, $g_{\pi}=\frac{i_{b}}{v_{b e}}$ and

$g_{e}=\frac{(1+\beta) i_{b}}{v_{e b}}=(1+\beta) \frac{i_{b}}{v_{e b}}=(1+\beta) g_{\pi}$, and $r_{\pi}=(1+\beta) r_{e}$,
$G_{L}=G_{C}+G_{L 1}$

\section{COMMON EMITTER AMPLIFIER}

To obtain the floating admittance matrix of the common emitter amplifier from the floating admittance matrix of the phase-splitter circuit of Eq (17), its $3^{\text {rd }}$ row and $3^{\text {rd }}$ column are added to its $4^{\text {th }}$ row and $4^{\text {th }}$ column. Now, deleting the original $3^{\text {rd }}$ row and $3^{\text {rd }}$ column and assigning the $4^{\text {th }}$ row and $4^{\text {th }}$ column as $3^{\text {rd }}$ row and $3^{\text {rd }}$ column yields the matrix;

$\left[\begin{array}{ccc}1 & 2 & 3 \\ g_{\pi}+g_{s}+G_{B} & 0 & -g_{\pi}-g_{s}-G_{B} \\ g_{m} & g_{o}+G_{L} & -g_{m}-g_{o}-G_{L} \\ -g_{\pi}-g_{m}-g_{s}-G_{R} & -g_{n}-G_{I} & g_{\pi}+g_{m}+g_{n}+g_{s}+G_{R}+G_{L}\end{array}\right]\left[\begin{array}{l}1 \\ 1 \\ 2 \\ 3\end{array}\right]$

This Eq (18) is the floating admittance matrix of Common Emitter Amplifier in hybrid- $\pi$ model shown in Fig. 7.

The voltage gain between its output terminals $2 \& 3$ and input terminals $1 \& 3$ of a common emitter amplifier in Fig. 7 results [10]-[17] as;

$\left.A_{v}\right|_{13} ^{23}=\operatorname{sgn}(2-3) \operatorname{sgn}(1-3)(-1)^{9} \frac{\left|Y_{23}^{13}\right|}{\left|Y_{13}^{13}\right|}$

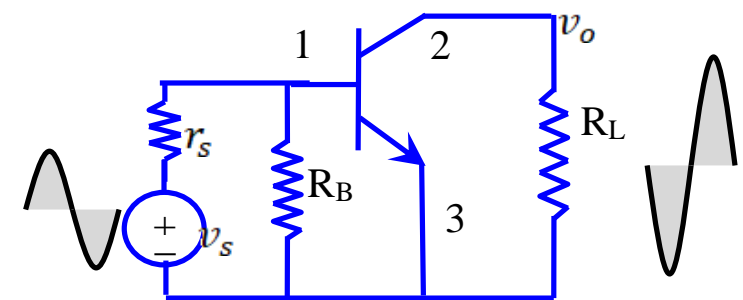


Equation (28) is the floating admittance matrix of the Common Collector Amplifier shown in Fig. 8. The voltage gain between output terminals $3 \& 2$ and input terminals $1 \& 2$ of a common collector amplifier in Fig. 8 results [10]-[14] as;

Fig. 7 Common Emitter Amplifier

$\left|Y_{23}^{13}\right|=g_{m},\left|Y_{13}^{13}\right|=g_{o}+G_{L}$

$\left.A_{v}\right|_{13} ^{23}=-\frac{g_{m}}{g_{o}+G_{L}}=-g_{m}\left(r_{o} \| R_{L}\right) \cong-g_{m} R_{L}$

The input resistance between terminals $1 \& 3$ of a common emitter amplifier in Fig. 7 results [10]-[13], [16]-[17]] as;

$$
\begin{aligned}
& R_{i(13)}=\frac{\left|Y_{13}^{13}\right|}{\left|Y_{3}^{3}\right|} g_{s}=0 \\
& \left|Y_{13}^{13}\right|=g_{o}+G_{L} \\
& \left|Y_{3}^{3}\right|_{g_{s}=0}=\left|\begin{array}{cc}
g_{\pi}+G_{B} & 0 \\
g_{m} & g_{0}+G_{L}
\end{array}\right| \\
& =\left(g_{\pi}+G_{B}\right)\left(g_{0}+G_{L}\right)=\left(g_{\pi}+G_{B}\right)\left(g_{o}+G_{L}\right) \\
& R_{i(13)}=\frac{\left|Y_{13}^{13}\right|}{\left|Y_{3}^{3}\right|_{g_{s}=0}}=\frac{g_{O}+G_{L}}{\left(g_{\pi}+G_{B}\right)\left(g_{O}+G_{L}\right)}=r_{\pi} \| R_{B} \cong r_{\pi}
\end{aligned}
$$

The output resistance between terminals $2 \& 3$ of common emitter amplifier in Fig. 7 results [10]-[17] as;

$$
\begin{aligned}
& R_{O(23)}=\frac{\left|Y_{23}^{23}\right|}{\left|Y_{3}^{3}\right|} \\
& \left|Y_{G_{L}}^{23}\right|=0 \\
& \left|Y_{3}^{3}\right|_{G_{L}=0}=\left(g_{s}+g_{B}+g_{B}\right) g_{O} \\
& R_{O(23)}=\frac{g_{\pi}+g_{s}+G_{B}}{\left(g_{\pi}+g_{s}+G_{B}\right) g_{O}}=\frac{1}{g_{O}}=r_{O}
\end{aligned}
$$

The current gain between output terminals $2 \& 3$ and input terminals $1 \& 3$ of common emitter amplifier in Fig. 7 results [10]-[12] as;

$$
\begin{gathered}
\left.A_{i}\right|_{13} ^{23}=\operatorname{sgn}(2-3) \operatorname{sgn}(1-3)(-1)^{9} \frac{\left|Y_{23}^{13}\right|}{\left|Y_{3}^{3}\right|} G_{L} \\
\begin{array}{c}
\left.A_{i}\right|_{13} ^{23}=-\frac{\left|Y_{23}^{13}\right|}{\left|Y_{3}^{3}\right|} G_{L}=-\frac{g_{m}}{\left(g_{\pi}+g_{s}+G_{B}\right)\left(g_{o}+G_{L}\right)} G_{L} \\
=-g_{m}\left(\frac{1}{g_{\pi}}\right)=-\beta
\end{array}
\end{gathered}
$$

The power gain between output terminals $2 \& 3$ and input terminals $1 \& 3$ of a common emitter amplifier in Fig. 7 results [10]-[11] as;

$$
\begin{aligned}
\left.A_{P}\right|_{13} ^{23} & =\left.\left.A_{v}\right|_{13} ^{23} x A_{i}\right|_{13} ^{23}=\left(-g_{m} R_{L}\right)(-\beta) \\
& =\beta g_{m} R_{L}
\end{aligned}
$$

\section{COMMON COLLECTOR AMPLIFIER}

To derive the floating admittance matrix of the common collector amplifier from the floating admittance matrix of the phase-splitter circuit of (17), its $2^{\text {nd }}$ row and $2^{\text {nd }}$ column are added to the $4^{\text {th }}$ row and $4^{\text {th }}$ column. Now, the original $2^{\text {nd }}$ row and $2^{\text {nd }}$ column are deleted after assigning $4^{\text {th }}$ row, and $4^{\text {th }}$ column as $2^{\text {nd }}$ row and $2^{\text {nd }}$ column yields the matrix as;

$\left[\begin{array}{ccc}1 & 3 & 2 \\ g_{\pi}+g_{s}+G_{B} & -g_{\pi} & -g_{s}-G_{B} \\ -g_{\pi}-g_{m} & g_{\pi}+g_{m}+g_{o}+G_{E} & -g_{o}-G_{E} \\ g_{m}-g_{s}-G_{R} & -g_{m}-g_{n}-G_{R} & g_{n}+g_{s}+G_{R}+G_{R}\end{array}\right]\left[\begin{array}{l}1 \\ 3 \\ 2\end{array}\right]$

$$
\begin{aligned}
\left.A_{v}\right|_{12} ^{32} & =\operatorname{sgn}(3-2) \operatorname{sgn}(1-2)(-1)^{8} \frac{\left|Y_{32}^{12}\right|}{\left|Y_{12}^{12}\right|} \\
\left|Y_{32}^{12}\right| & =-g_{\pi}-g_{m}=-g_{\pi}(1+\beta) \\
\left|Y_{12}^{12}\right| & =g_{\pi}+g_{m}+g_{o}+G_{E} \cong g_{\pi}+g_{m}+G_{E} \\
\left.A_{v}\right|_{12} ^{32} & =-\frac{-(1+\beta) g_{\pi}}{(1+\beta) g_{\pi}+G_{E}} \frac{R_{E}}{R_{E}+\frac{r_{\pi}}{(1+\beta)}}=\frac{R_{E}}{R_{E}+\frac{(1+\beta) r_{e}}{(1+\beta)}} \\
& =\frac{R_{E}}{R_{E}+r_{e}} \cong 1
\end{aligned}
$$

Equation (30) defines the voltage gain of the emitter follower, i.e., common collector amplifier.

The current gain between output terminals $3 \& 2$ and input terminals $1 \& 2$ of a common collector amplifier in Fig. 8 results [10]-[11] as;

$$
\left.A_{i}\right|_{12} ^{32}=\operatorname{sgn}(3-2) \operatorname{sgn}(1-2)(-1)^{8} \frac{\left|Y_{32}^{12}\right|}{\left|Y_{2}^{2}\right|} G_{E}
$$

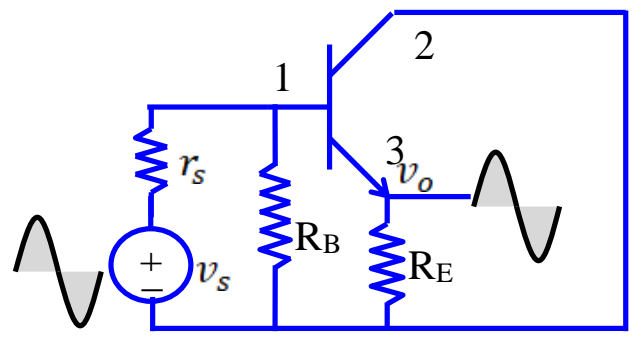

Fig. 8 Common Collector Amplifier

$$
\begin{aligned}
& \left|Y_{32}^{12}\right|=-g_{\pi}-g_{m}=-(1+\beta) g_{\pi} \\
& \left|Y_{2}^{2}\right|=\left|\begin{array}{cc}
g_{\pi} & -g_{\pi} \\
-g_{\pi}-g_{m} & g_{\pi}+g_{m}+g_{o}+G_{E}
\end{array}\right| \\
& =\left|\begin{array}{cc}
g_{\pi} & 0 \\
-g_{\pi}-g_{m} & g_{o}+G_{E}
\end{array}\right|=g_{\pi} G_{E} \\
& \left.A_{i}\right|_{12} ^{32}=-\frac{-(1+\beta) g_{\pi}}{g_{\pi} G_{E}} G_{E}=1+\beta
\end{aligned}
$$

Equation Eq (32) is the ideal current gain of the emitter follower circuit.

The input resistance between terminals $1 \& 2$ in Fig. 8 using Eq (28) results [10]-[13], [16]-[17]] as;

$$
\begin{gathered}
R_{i(12)}=\frac{\left|Y_{12}^{12}\right|}{\left|Y_{2}^{2}\right|} g_{s=0} \\
\left|Y_{12}^{12}\right|=(1+\beta) g_{\pi}+g_{o}+G_{E} \\
\left|Y_{2}^{2}\right|_{g_{s}=0}=\mid \begin{array}{cc}
g_{\pi} & -g_{\pi} \\
-g_{\pi}-g_{m} & g_{\pi}+g_{m}+g_{o}+G_{E} \mid \\
=\left|\begin{array}{cc}
g_{\pi} & 0 \\
-g_{\pi}-g_{m} & g_{o}+G_{E}
\end{array}\right|=g_{\pi}\left(g_{o}+G_{E}\right) \\
R_{i(12)}=\frac{(1+\beta) g_{\pi}+g_{o}+G_{E}}{g_{\pi}\left(g_{o}+G_{E}\right)}=\frac{(1+\beta) g_{\pi}+G_{E}}{g_{\pi} G_{E}} \\
=r_{\pi}+(1+\beta) R_{E}
\end{array}
\end{gathered}
$$


The output resistance between terminals $3 \& 2$ of a common collector amplifier in Fig. 8 results [10]-[13], [16][17]] as;

$$
\begin{gathered}
R_{O(32)}=\frac{\left|Y_{32}^{32}\right|}{\left|Y_{2}^{2}\right|} G_{E}=0 \\
\left|Y_{32}^{32}\right|=g_{\pi}+g_{s}+G_{B}=g_{\pi}+g_{s} \\
\left|Y_{2}^{2}\right|_{G_{E}=0}=\left|\begin{array}{cc}
g_{\pi}+g_{s} & -g_{\pi} \\
-g_{\pi}-g_{m} & g_{\pi}+g_{m}+g_{o}
\end{array}\right| \\
=\left|\begin{array}{cc}
g_{\pi}+g_{s} & g_{s} \\
-g_{\pi}-g_{m} & g_{o}
\end{array}\right|=\left(g_{\pi}+g_{s}\right) g_{o}+(1+\beta) g_{\pi} g_{s} \\
R_{O(32)}=\frac{g_{\pi}+g_{s}}{\left(g_{\pi}+g_{s}\right) g_{O}+(1+\beta) g_{\pi} g_{s}} \\
G_{O(32)}=\frac{1}{R_{O(32)}}=\frac{\left(g_{\pi}+g_{s}\right) g_{O}+(1+\beta) g_{\pi} g_{s}}{g_{\pi}+g_{s}} \\
=g_{O}+\frac{(1+\beta) g_{\pi} g_{s}}{g_{\pi}+g_{s}}=g_{O}+\frac{(1+\beta)}{r_{\pi}+r_{s}} \\
R_{O(32)}=\frac{r_{\pi}+r_{s}}{1+\beta}
\end{gathered}
$$

Power gain $=\left.\left.A_{v}\right|_{12} ^{32} x A_{i}\right|_{12} ^{32}=\left(\frac{R_{E}}{R_{E}+r_{\varepsilon}}\right)(1+\beta)$

\section{COMMON BASE AMPLIFIER}

To derive the circuit and floating admittance matrix of a common base amplifier in hybrid- $\pi$ model from the circuit of a phase-splitter shown in Fig. 1 and its (17), its $1^{\text {st }}$ row and $1^{\text {st }}$ column are added to the $4^{\text {th }}$ row and $4^{\text {th }}$ column. Now, the original $1^{\text {st }}$ row and $1^{\text {st }}$ column are deleted, and $4^{\text {th }}$ row and $4^{\text {th }}$ column are assigned $1^{\text {st }}$ row and $1^{\text {st }}$ column with $G_{E}=G_{E}+g_{s}$ yields;

$$
\left[\begin{array}{ccc}
2 & 3 & 1 \\
g_{o}+G_{L} & -g_{m}-g_{o} & g_{m}-G_{L} \\
-g_{o} & g_{m}+g_{\pi}+g_{o}+g_{s}+G_{E} & -g_{m}-g_{\pi}-g_{s}-G_{E} \\
-G_{L} & -g_{\pi}-g_{s}-G_{E} & g_{\pi}+g_{s}+G_{E}+G_{L}
\end{array}\right]\left[\begin{array}{l}
2 \\
2 \\
3 \\
1
\end{array}\right]
$$

The circuit of Fig. 9 of a common base amplifier results from (38). The BJT base terminal is common to both input and output sides in Fig. 9, and it is called a common base amplifier.

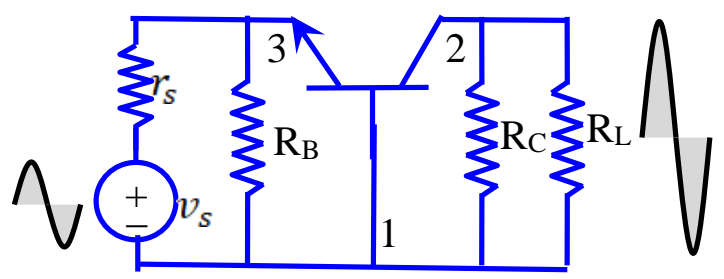

Fig. 9 Common Base Amplifier

The voltage gain between its output terminals $2 \& 1$ and input terminals $3 \& 1$ of a common base amplifier in Fig. 9 results [10]-[14] as;
$\left.A_{v}\right|_{31} ^{21}=\operatorname{sgn}(2-1) \operatorname{sgn}(3-1)(-1)^{7} \frac{\left|Y_{21}^{31}\right|}{\left|Y_{31}^{31}\right|}$

$\left|Y_{21}^{31}\right|=-g_{m}-g_{o},\left|Y_{31}^{31}\right|=g_{o}+G_{L}$

$\left.A_{v}\right|_{31} ^{21}=-\frac{-g_{m}-\sharp 0}{\sharp 0+G_{L}}=\frac{g_{m}}{G_{L}}=g_{m} R_{L}$

The current gain between its output terminals $2 \& 1$ and input terminals $3 \& 1$ of a common base amplifier in Fig. 9 using (38) results [10]-[11] as;

$$
\begin{aligned}
& \left.A_{i}\right|_{31} ^{21}=\operatorname{sgn}(2-1) \operatorname{sgn}(3-1)(-1)^{7} \frac{\left|Y_{21}^{31}\right|}{\left|Y_{1}^{1}\right|} G_{L} \\
& \left|Y_{1}^{1}\right|=\left|\begin{array}{cc}
\theta_{o}+G_{L} & -g_{m}-\uplus_{0} \\
-f_{\Delta} & (1+\beta) g_{\pi}+\sharp_{0}+G_{s}
\end{array}\right| \\
& =(1+\beta) g_{\pi} G_{L} \\
& \left.A_{i}\right|_{31} ^{21}=-\frac{-\beta g_{\pi}-\theta_{\ominus}}{(1+\beta) g_{\pi} G_{L}} G_{L}=\frac{\beta}{(1+\beta)}=\alpha
\end{aligned}
$$

The input resistance between terminals $3 \& 1$ of a common base amplifier in Fig. 9 results [10]-[13], [16]-[17]] as;

$$
\begin{aligned}
& R_{i}=R_{i(31)}=\frac{\left|Y_{31}^{31}\right|}{\left|Y_{1}^{1}\right|} g_{s=0} \\
& \left|Y_{31}^{31}\right|=g_{0}+G_{L}
\end{aligned}
$$

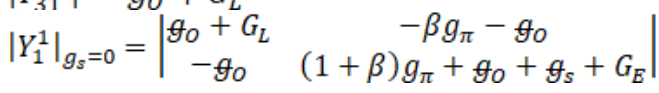

$$
\begin{aligned}
& =\left\{(1+\beta) g_{\pi}+G_{E}\right\} G_{L}=\left\{r_{\pi}+(1+\beta) R_{E}\right\} G_{L} g_{\pi} G_{E} \\
& R_{i}=R_{i(31)}=\frac{\bigoplus_{o}+G_{L}}{\left\{r_{\pi}+(1+\beta) R_{E}\right\} G_{L} g_{\pi} G_{E}} \\
& =\frac{r_{\pi}}{(1+\beta)}=\frac{(1+\beta) r_{e}}{(1+\beta)}=r_{e}=\frac{1}{g_{m}}
\end{aligned}
$$

The output resistance between terminals $2 \& 1$ of a common base amplifier in Fig. 9 results [10]-[13], [16]-[17]] as;

$$
\begin{aligned}
& R_{O(21)}=\frac{\left|Y_{21}^{21}\right|}{\left|Y_{1}^{1}\right|}{ }_{G_{L}=0} \\
& \left|Y_{21}^{21}\right|=(1+\beta) g_{\pi}+y_{0}+y_{s}+G_{E} \\
& =\left\{r_{\pi}+(1+\beta) R_{E}\right\} g_{\pi} G_{E} \\
& \left|Y_{1}^{1}\right|_{G_{\mathrm{L}}=0}=\left|\begin{array}{cc}
g_{o} & -g_{m}-g_{0} \\
-g_{o} & g_{m}+g_{\pi}+g_{o}+g_{s}+G_{E}
\end{array}\right| \\
& =\left|\begin{array}{cc}
g_{o} & -g_{m}-g_{\pi} \\
0 & g_{\pi}+g_{s}+G_{E}
\end{array}\right| \\
& R_{O}=R_{O(21)}=\frac{\left\{r_{\pi}+(1+\beta) R_{E}\right\} g_{\pi} G_{E}}{g_{O}\left(g_{\pi}+G_{E}\right)}=\frac{\left\{r_{\pi}+(1+\beta) R_{E}\right\}}{g_{O}\left(r_{\pi}+R_{E}\right)} \\
& =\frac{r_{\pi}+(1+\beta) R_{E}}{g_{O} R_{E}}=\frac{(1+\beta) R_{E}}{g_{O} R_{E}}=(1+\beta) r_{O}
\end{aligned}
$$

If we look at the output resistance through $R_{I}^{\prime}$, then

$$
R_{O(21)}=(1+\beta) r_{O}|| R_{C} \cong R_{C}
$$

Power gain $=A_{p}=A_{v} x A_{i}=g_{m} \alpha R_{L}$ 


\section{CONCLUSIONS}

The floating admittance matrix method of analysis is very simple. Anybody with little knowledge of electronics and the matrix maneuvering process can quickly analyze the circuit. Additionally, the floating admittance matrix's zero-sum property provides a self-check before further analyzing the circuit. The method does not require a small-signal equivalent circuit. All transfer functions of any circuit use only cofactors ratios so that calculation can be accurate without any approximation.

\section{REFERENCES}

[1] Chirlian P., Electronic Circuits-Physical Principles Analysis and Design, McGraw-Hill.

[2] Sedra A. S., and K. C. Smith, 2002, "Microelectronic Circuits," $7^{\text {th }}$ Edition, Oxford University Press, New York.

[3] Neamen, "Semiconductor Physics and Devices-Basic Principles," McGraw-Hill, 2010.

[4] Giacoletto L. J., "Study of pnp Alloy Junction Transistor from DC through Medium frequencies," RCA Rev., vol. 15, no.4, pp. 506-562, December, 1954.

[5] Mitra, S. K., "Analysis and Synthesis of Linear Active Networks," John Wiley.

[6] Ramey R. L. and White E. J., "Matrices and Computers in Electronic Circuit Analysis," McGrawHill.

[7] Haigh, David G., and Paul M. Radmore. "Admittance matrix models for the nullor using limit variables and their application to circuit design", IEEE Transactions on Circuits and Systems I: Regular Papers 53, no. 10 (2006): 2214-2223.

[8] Vago, I., and E. Hollos. "Two-port models with nullators and norators", Periodica Polytechnica Electrical Engineering 17, no. 4 (1973): 301-309.

[9] Kumar, Pragati, and Senani, Raj; "Improved grounded-capacitor SRCO using only a single PFTFN", Analog Integrated Circuits and Signal Processing 50, no. 2 (2007): 147-149.

[10] Rao, DBSJ Prasada, Singh, B. P., "Unified Approach to Electronics Circuit Analysis", IJEEE, Vol., pp.276-285, July 1978, ISSN: 0020720.

[11] Singh, Meena, Roy, S K, Singh, B P Singh and Shukla, S N "Mathematical Modeling of Electronic Devices and its Integration;" Proceedings of National Seminar on Recent Advances on Information Technology, Organized by IIT (ISM) Dhanbad University, and Proceedings Published by Allied Publishers Pvt. Ltd. New Delhi, pp.494-502, Feb. 67, 2009.

[12] Singh and Pragati P, "Floating Admittance Matrix Modeling Approach to BJT," IJRSI, Vol. VI, Issue X, October 2019.

[13] Roy, S K, Singh, M, Sharma, K K, Bhargava, Cherry, Singh, B P, "Mathematical Modelling of Semiconductor Devices and circuits: A Review;" $3^{\text {rd }}$ International Conference on Intelligent Circuits and
Systems (ICICS), June 26-27, 2020, Lovely Professional University, (Phagwara) Punjab.

[14]Roy, S K, Singh, M, Sharma, K K, Bhargava, Cherry, Singh, B P, "Mathematical Modelling of Simple Passive RC Filters Using Floating Admittance Technique," 2020 IEEE International Conference for Innovation in Technology (INOCON-20), Bengaluru, India. Nov 6-8, 2020.

[15] S. K. Roy, M. Singh, K. K. Sharma, B. Cherry, and B. P. Singh, "Mathematical Modelling of Simple Passive RC Filters Using Floating Admittance Technique," 2020 IEEE International Conference for Innovation in Technology (INOCON-20), Bengaluru, India. Nov 6-8, pp. 1-6, 2020.

[16] Sanjay Kumar Roy, Kamal Kumar Sharma, Cherry Bhargava, Brahmadeo Prasad Singh, Mathematical Modelling and Simulation of Band Pass Filters using the Floating Admittance Matrix Method, WSEAS Transactions on Circuits and Systems, Vol. 20, pp. 208-214, 2021 DOI: 10.37394/23201.2021.20.24.

[17] Sanjay Kumar Roy, Kamal Kumar Sharma, and Brahmadeo Prasad Singh, Unique Analysis Approach to Bridge-T Network using Floating Admittance Matrix Method, International Journal of Circuits, Systems and Signal Processing Volume 15, pp.12971304, 2021 DOI: 10.46300/9106.2021.15.140.

\section{Creative Commons Attribution License 4.0 (Attribution 4.0 International, CC BY 4.0)}

This article is published under the terms of the Creative Commons Attribution License 4.0 https://creativecommons.org/licenses/by/4.0/deed.en_US 